Research Article

\title{
Upregulation of ECT2 Predicts Adverse Clinical Outcomes and Increases 5-Fluorouracil Resistance in Gastric Cancer Patients
}

\author{
Hua Zhang $\mathbb{D}^{1}$ ' Yuan Geng $\mathbb{D},{ }^{2}$ Chunhui Sun $\mathbb{D}^{3},{ }^{3}$ and Jin Yu $\mathbb{D}^{4}$ \\ ${ }^{1}$ Department of Pharmacy, Yantaishan Hospital, Yantai 264000, Shandong, China \\ ${ }^{2}$ Department of Obstetrics, Medical Insurance Office, Qingdao Municipal Hospital, Qingdao 266000, Shandong, China \\ ${ }^{3}$ Department of Hepatobiliary Surgery, The Third People's Hospital of Qingdao, Qingdao 266041, Shandong, China \\ ${ }^{4}$ Department of Oncology, Jinan Central Hospital, Cheeloo College of Medicine, Shandong University, Jinan 250013, \\ Shandong, China
}

Correspondence should be addressed to Jin Yu; guaojingq@163.com

Received 1 July 2021; Revised 15 July 2021; Accepted 16 July 2021; Published 28 July 2021

Academic Editor: Muhammad Wasim Khan

Copyright (C) 2021 Hua Zhang et al. This is an open access article distributed under the Creative Commons Attribution License, which permits unrestricted use, distribution, and reproduction in any medium, provided the original work is properly cited.

Background. The abnormal expression and prognosis prediction of epithelial cell transforming sequence 2 (ECT2) in gastric cancer (GC) has been reported. However, the effect of ECT2 on 5-fluorouracil (5-Fu) resistance in GC is unclear. This research aims to solve the abovementioned problems. Methods. Gene expression was detected by RT-qPCR and Western blot analysis. Cell viability was evaluated by the colony formation assay, MTT assay, and flow cytometric analysis. Transwell and wound healing assays were used to detect cell metastasis. Results. Upregulation of ECT2 was found in stomach adenocarcinoma (STAD) and GC tissues. In addition, high ECT2 expression can predict adverse clinical outcomes in GC patients. More importantly, ECT2 knockdown weakened the resistance of 5-FU in GC cells. ECT2 silencing reduced the cell migratory and invasive abilities of GC cells treated with 5-FU. We also found that downregulation of ECT2 increased 5-FU sensitivity in GC cells by downregulating $\mathrm{P}$-gp, MRP1, and Bcl-2. Conclusion. Upregulation of ECT2 can predict adverse clinical outcomes and increase 5-FU resistance in GC patients.

\section{Introduction}

Gastric cancer (GC) is one of the cancers with the highest incidence in the world [1]. Various gastric diseases, Helicobacter pylori (Hp) infection, poor diet, environment, and genetics can cause GC [2]. The incidence of GC increases significantly with age, and the peak age of onset is 50-80 years [3]. China is a high incidence area of GC. GC accounts for nearly a quarter of cancer deaths in China [4]. Moreover, the early diagnosis rate of GC is low, about $10 \%$. Most patients with GC are in the middle and advanced stages at the time of diagnosis. The 5 -year survival rate is about $7-34 \%$ [5]. Recently, more and more methods are being used to treat GC, such as radiotherapy, chemotherapy, cell membrane-derived biomimetic nanotechnology, and cancer nanomedicine $[6,7]$. Preoperative chemotherapy can shrink the tumor and increase the chance of radical surgery and cure. However, the resistance of cancer cells to chemotherapy drugs can lead to chemotherapy failure [8]. Therefore, exploring new methods to weaken the drug resistance of cancer cells is of great significance to improve the success rate of chemotherapy.

Commonly used chemotherapy drugs are 5-fluorouracil (5-Fu), tegafur, mitomycin, doxorubicin, paclitaxel, cisplatin, or carboplatin $[9,10]$. Many studies have shown that no-coding RNAs or genes can affect the resistance of cancer cells. For example, TRIM37 increased the resistance to CDDP in GC [11]. MiR-95-3p acted as a contributing factor for cisplatin resistance in human GC cells by targeting EMP1/PI3K/AKT signaling [12]. The exosomal miR-223 enhanced the resistance of doxorubicin in GC [13]. In addition, it was found that upregulation of KLF17 increased 5Fu sensitivity in GC cells [14]. Here, the effect of ECT2 on 5$\mathrm{Fu}$ resistance was investigated in GC. 
ECT2 has been found to be involved in the development of various human cancers. Increased expression of ECT2 has been found in many malignant tumors, such as breast cancer [15], cholangiocarcinoma [16], and hepatocellular carcinoma [17]. Functionally, the ECT2/ PSMD14/PTTG1 axis promoted the proliferation of gliomas by stabilizing E2F1 [18]. The inhibition of ECT2 induced by small interfering RNA suppressed the progression of osteosarcoma [19]. ECT2 overexpression also promoted the polarization of tumor-associated macrophages in hepatocellular carcinoma [20]. More importantly, upregulation of ECT2 has been found in stomach adenocarcinoma (STAD) and GC tissues [21]. However, the effect of ECT2 on 5-Fu resistance in GC cells has not been reported yet.

Here, the expression level of ECT2 was first detected in GC. The correlation between the expression of ECT2 and the clinical outcome of GC patients was also confirmed. In addition, we have also explored how ECT2 affects $5-\mathrm{Fu}$ resistant in GC cells. Our research may provide a new way of thinking about weakening 5 -Fu resistance.

\section{Materials and Methods}

2.1. Patients. Tissue samples of 66GC patients were collected from Jinan Central Hospital between July 2018 and July 2020. The content of the study is informed to everyone, and we have obtained their informed consents. All participants received only surgical treatment. Our study was approved by the Institutional Ethics Committee of Jinan Central Hospital.

2.2. Bioinformatics Analysis. The expression and prognosis of ECT2 in GC patients were analyzed by box plots and survival plots in the GEPIA database (http://gepia.cancerpku.cn/).

2.3. Cell Culture and Transfection. Normal gastric mucosal cell lines GES-1 and AGS and NCI-N87GC cells were purchased from ATCC (Manassas, VA, USA). The above cells were cultured in RPMI-1640 medium (Gibco, USA) containing $10 \% \mathrm{FBS}\left(37^{\circ} \mathrm{C}, 5 \% \mathrm{CO}_{2}\right)$. ECT2 siRNA (siECT2) and si-control (si-NC) were purchased from GenePharma (Shanghai, China). Lipofectamine 2000 (Invitrogen, Carlsbad, USA) was used for cell transfection according to the manufacturer's instructions.

2.4. RT-qPCR. The total RNA was extracted with TRIzol reagent (Thermo Fisher Scientific). PrimeScript-RT Kit (Madison, WI, USA) was used to synthesize complementary DNA (cDNA). RT-qPCR was performed using SYBR ${ }^{\circledR}$ Premix-Ex-Taq ${ }^{\mathrm{TM}}$ (Takara, TX, USA). The internal reference is GAPDH. The specific primer pairs were as follows: ECT2, sense: $5^{\prime}$-ACT ACT GGG AGG ACT AGC TTG-3' and antisense: $5^{\prime}$-CAC TCT TGT TTC AAT CTG AGG CA-3'; GAPDH, sense: $5^{\prime}$-GGA GCG AGA TCC CTC CAA AAT- $3^{\prime}$ and antisense: $5^{\prime}$-GGC TGT TGT CAT ACT TCT CAT GG$3^{\prime}$. The $2^{-\Delta \Delta c t}$ method was used to measure the relative expression of ECT2.

2.5. Western Blot Analysis. Protein samples were isolated by using RIPA lysis buffer (Beyotime Biotechnology). Then, the protein samples were separated by $10 \%$ SDSPAGE. After transferring to the PVDF membrane, the protein was blocked with $5 \%$ skimmed milk. Next, the protein samples were incubated with Bax, Bcl-2, MRP1, P-gp, GST- $\pi$, and GAPDH primary antibodies (Abcam, Cambridge, MA, USA) at $4^{\circ} \mathrm{C}$ overnight. After washing 3 times, the protein samples were incubated with the corresponding secondary antibody (Abcam, USA) for $2 \mathrm{~h}$. Finally, the ECL detection system (Thermo Fisher Scientific, Inc.) was used to visualize the blots. The relative level of protein expression was analyzed using ImagePro plus software (version 6.0; Media Cybernetics Inc., Rockville, MD, USA) and is represented as the density ratio versus GAPDH.

2.6. Cell Viability Assay. The half maximal inhibitory concentration (IC50) of AGS on 5-FU was detected by the MTT assay. AGS cells were cultured in 96-well plates for $24 \mathrm{~h}$. Next, different concentrations of 5-FU were added to treat the AGS cells for $48 \mathrm{~h}$. After that, MTT solution was added to incubate the cells at $37^{\circ} \mathrm{C}$ for $4 \mathrm{~h}$. Finally, the absorbance value $(\mathrm{OD}=490 \mathrm{~nm})$ was measured.

2.7. Colony Formation Assay. The transfected AGS cells were cultured in 6-well plates (1000 cells/well) for three days. Then, 5-FU was added to treat the cells for 10 days. Next, the cells were stained with $0.1 \%$ crystal violet. Finally, colonies were observed by a light microscope.

2.8. Flow Cytometric Analysis. The transfected AGS cells were suspended in Annexin-binding buffer and harvested. Then, the cells were stained with Annexin V/FITC and PI solution (KeyGEN Biotech, Nanjing, China) and incubated in the dark for $15 \mathrm{~min}$ at room temperature. Finally, flow cytometry analysis was used to assess cell apoptosis.

2.9. Wound Healing Assay. AGS cells $\left(1 \times 10^{3}\right.$ cells/well $)$ were cultured in a 6 -well plate for $24 \mathrm{~h}$. After reaching $90 \%$ confluence, scratches were generated by a $200 \mu \mathrm{l}$ pipette tip. A light microscope was used to evaluate the wound width at 0 and $24 \mathrm{~h}$. Images were captured by THUNDER Imagers (Leica Microsystems). Wound distance was quantified by ImageJ Software version 1.6 (National Institutes of Health, Bethesda, MD, USA).

2.10. Transwell Assay. Cell migration and invasion were analyzed by performing transwell assays. Transwell chambers ( $8 \mu \mathrm{m}$ pore size; Millipore) were applied to evaluate cell migration and invasion. For the invasion assay, AGS cells 
$\left(4 \times 10^{3}\right.$ cells/well $)$ were added into the upper chamber, which was precoated with Matrigel $(3.9 \mathrm{ug} / \mathrm{ul})$. For the migration assay, AGS cells $\left(4 \times 10^{3}\right.$ cells/well) were added into the upper chamber, which was not precoated with Matrigel. Lower chamber was added with RPMI-1640 medium (10\% FBS). Following incubation for $24 \mathrm{~h}$ at $37^{\circ} \mathrm{C}$ with $5 \% \mathrm{CO}_{2}$, the migrated and invasive cells were stained with $0.1 \%$ crystal violet. The number of moved cells was observed under a light microscope.

2.11. Statistical Analysis. GraphPad Prism 6 or SPSS 21.0 is used to analyze experimental data. The results are shown as mean \pm SD. The difference among groups was analyzed by using the chi-squared test or one-way ANOVA with the Bonferroni post hoc test. Significant difference means $p<0.05$.

\section{Results}

3.1. Upregulation of ECT2 Predicts Adverse Clinical Outcomes in GC Patients. First, the expression level of ECT2 was analyzed in the GEPIA database (http://gepia.cancer-pku. $\mathrm{cn} /$ ). Compared with the control, the expression of ECT2 was increased in stomach adenocarcinoma (STAD) tissues $(p<0.05$, Figure 1(a)). Consistently, upregulation of ECT2 in GC tissues was also found in GC samples $(p<0.05$, Figure 1(b)). In addition, the GEPIA database showed that GC patients with high ECT2 expression had a reduced overall survival (OS) rate $(p<0.05$, Figure $1(\mathrm{c}))$. We also found that the high expression of ECT2 was related to lymph node metastasis and TNM stage in GC patients $(p<0.05$, Table 1). These findings imply that ECT2 may affect the development of GC.

3.2. Knockdown of ECT2 Enhances 5-FU Sensitivity in GC Cells. Next, the expression of ECT2 in GC cells was measured. Compared with GES-1, ECT2 expression was increased in AGS and NCI-N87GC cells $(p<0.05$, Figure $2(a))$. In order to verify the effect of ECT2 on the sensitivity of 5-FU in GC cells, si-ECT2 or si-NC was transfected into AGS cells. Compared with the si-NC group, si-ECT2 reduced the expression of ECT2 in AGS cells $(p<0.01$, Figure $2(\mathrm{~b}))$. In addition, the downregulation of ECT2 enhanced the sensitivity of 5 -FU in AGS cells $(p<0.01$, Figure $2(\mathrm{c}))$. The IC50 value of the siECT2 group was higher than that of the si-NC group $(p<0.05$, Figure $2(\mathrm{~d}))$. At the same time, the colony formation assay also showed that knockdown of ECT2 weakened the cloning ability of AGS cells treated with 5-FU $(p<0.05$, Figure 2(e)). The apoptosis of AGS cells treated with 5-FU was promoted by downregulation of ECT2 $(p<0.05$, Figure 2(f)). In short, ECT2 knockdown weakens 5 -FU resistance in GC cells.

3.3. ECT2 Silencing Inhibits the Metastasis of GC Cells Treated by 5-FU. In order to further explore the role of ECT2 in the development of GC, the effect of ECT2 on the metastasis of
5-FU-treated AGS cell was also investigated. Both the wound healing assay and the transwell assay showed that ECT2 downregulation significantly inhibited cell migration in AGS cells treated with 5-FU $(p<0.05$, Figures 3(a) and 3(b)). Compared with the si-NC group, cell invasion was also suppressed by ECT2 silencing in AGS cells treated with 5-FU $(p<0.01$, Figure 3(c)). Briefly, ECT2 silencing reduced the migratory and invasive ability of 5-FU-treated GC cells.

3.4. ECT2 Downregulation Increases the 5-FU Sensitivity of GC Cells by Inhibiting P-gp, MRP1, and Bcl-2. Finally, the effect of ECT2 on P-gp, MRP1, and GST- $\pi$ related to drug resistance was investigated to explore the regulatory mechanism of ECT2 on 5-FU sensitivity of GC cells. We found that the expression of P-gp and MRP1 in the si-ECT2 group was significantly reduced ( $p<0.05$, Figure $4(\mathrm{a}))$. However, ECT2 has little effect on GST- $\pi$ expression. In addition, we also explored how ECT2 regulates the apoptosis-related proteins Bcl-2 and Bax. Downregulation of ECT2 reduced Bcl-2 expression and enhanced Bax expression in AGS cells treated with 5-FU $(p<0.05$, Figure 4(b)). Taken together, ECT2 affects the 5-FU sensitivity of GC cells by regulating cell viability, metastasis, and apoptosis-related proteins (Figure 5).

\section{Discussion}

Recently, important functions of ECT2 have been discovered in many human diseases. In addition, the effect of ECT2 on chemoresistance has also been investigated in previous studies. For example, ECT2 can regulate the growth of triple-negative breast cancer cells through the intervention of paclitaxel [22]. In this study, the effect of ECT2 on 5-FU resistance was explored in GC cells. ECT2 expression was increased in STAD and GC tissues. Previous study also proposed the upregulation of ECT2 in STAD and GC tissues [21]. In addition, high ECT2 expression can predict adverse clinical outcomes in GC patients. More importantly, ECT2 knockdown weakened the resistance of 5 -FU in GC cells. ECT2 silencing reduced the migratory and invasive ability of GC cells treated with 5-FU. The above findings reveal that ECT2 may act as a tumor promoter in the progression of GC and increase 5-Fu resistance in GC patients.

Consistent with our results, Yan Chen et al. also found the upregulation and carcinogenic effects of ECT2 in GC [23]. In addition, upregulation of ECT2 also related to the poor prognosis of GC patients [24]. The same result is also found in our research. Besides that, it has been found that upregulation of ECT2 can predict adverse clinical outcomes in GC [25]. The histologic differentiation, TNM stages, and lymph node metastasis were related to ECT2 expression in GC patients [26]. This study also demonstrated the same conclusion. Functionally, upregulation of ECT2 promoted the tumor progression of renal cell carcinoma [27]. Overexpression of ECT2 also promoted the growth and metastasis of pancreatic cancer cells [28]. In this study, ECT2 silencing 


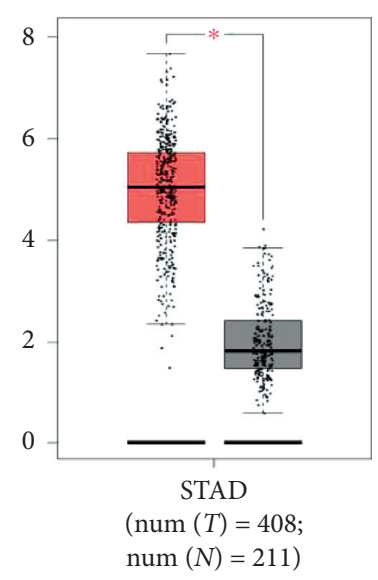

(a)

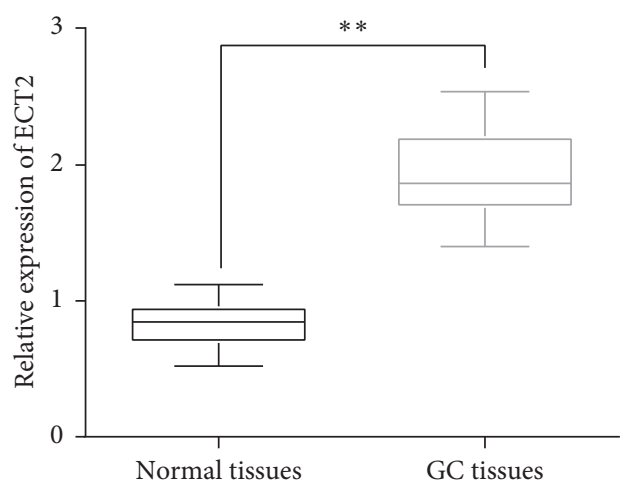

(b)

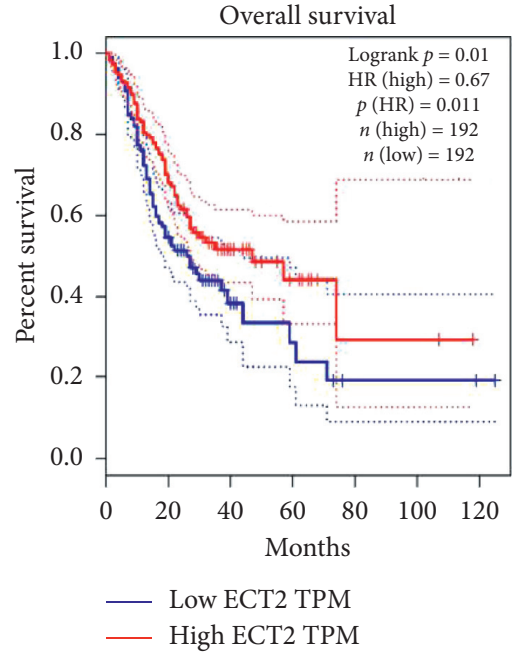

(c)

FIGURE 1: Upregulation of ECT2 predicting adverse clinical outcomes in GC patients. (a) ECT2 mRNA expression in STAD tissues $(n=408)$ and normal tissues $(n=211)$ analyzed in the GEPIA database. (b) ECT2 expression in 66GC tissues and adjacent normal tissues $(n=66)$. (c) OS rate was compared in GC patients with low or high ECT2 expression $(n=192) .{ }^{*} P<0.05 .{ }^{* *} P<0.01$.

TABLE 1: Relationship between ECT2 expression and clinic-pathological characteristics of GC patients.

\begin{tabular}{|c|c|c|c|c|}
\hline \multirow{2}{*}{ Characteristics } & \multirow{2}{*}{ Cases } & \multicolumn{2}{|c|}{ ECT2 } & \multirow{2}{*}{$P$ value } \\
\hline & & High & Low & \\
\hline \multicolumn{5}{|l|}{ Age (years) } \\
\hline$\geq 55$ & 30 & 18 & 12 & 0.083 \\
\hline$<55$ & 36 & 30 & 6 & \\
\hline \multicolumn{5}{|l|}{ Gender } \\
\hline Male & 38 & 28 & 10 & 0.124 \\
\hline Female & 28 & 20 & 8 & \\
\hline \multicolumn{5}{|l|}{ Tumor size (mm) } \\
\hline$\leq 5.0$ & 26 & 18 & 8 & 0.095 \\
\hline$>5.0$ & 40 & 30 & 10 & \\
\hline \multicolumn{5}{|l|}{ Differentiation } \\
\hline Well/moderate & 22 & 16 & 6 & 0.064 \\
\hline Poor & 44 & 32 & 12 & \\
\hline \multicolumn{5}{|c|}{ Lymph node metastasis } \\
\hline Yes & 42 & 30 & 12 & $0.042^{*}$ \\
\hline No & 24 & 18 & 6 & \\
\hline \multicolumn{5}{|l|}{ TNM stage } \\
\hline I-II & 22 & 15 & 7 & $0.024^{*}$ \\
\hline III-IV & 44 & 33 & 22 & \\
\hline
\end{tabular}

restrained the proliferation and metastasis of 5-FUtreated GC cells. These findings have not been found in previous studies.

In addition, we also found that downregulation of ECT2 increased the 5-FU sensitivity of GC cells by inhibiting Bcl-2, MRP1, and P-gp. P-gp can induce drug resistance by transporting drugs outside the cell [29]. MRP1 is an important gene that triggers cell resistance [30]. Apoptosis is not only related to tumor progression but also correlated with chemotherapy resistance. Among them, Bcl-2 is an antiapoptotic protein, and Bax is a proapoptotic protein [31]. Here, downregulation of ECT2 reduced the expression of Bcl-2, MRP1, and P-gp in GC cells. On the other hand, these results prove that ECT2 can increase 5-FU sensitivity in GC cells. However, our conclusion has not been verified in in vivo experiment. The function of ECT2 will be explored in vivo in the future. 


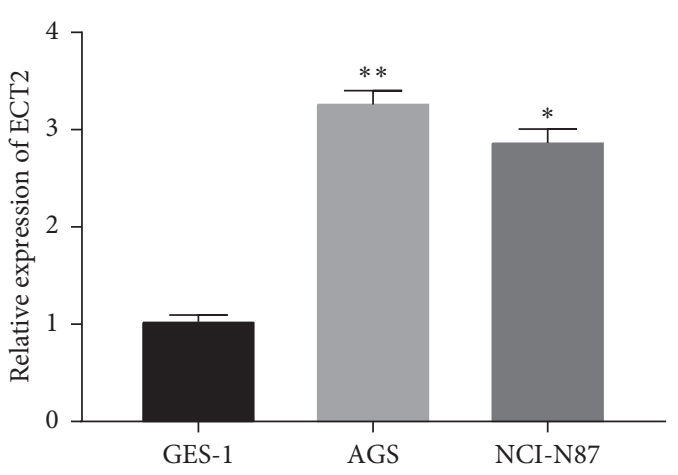

(a)

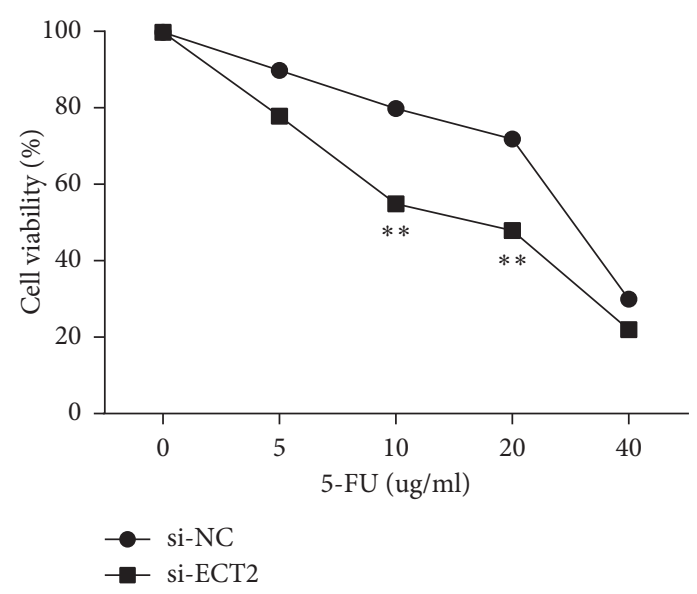

(c)

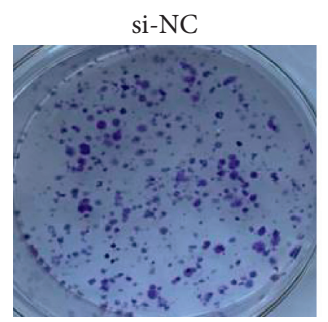

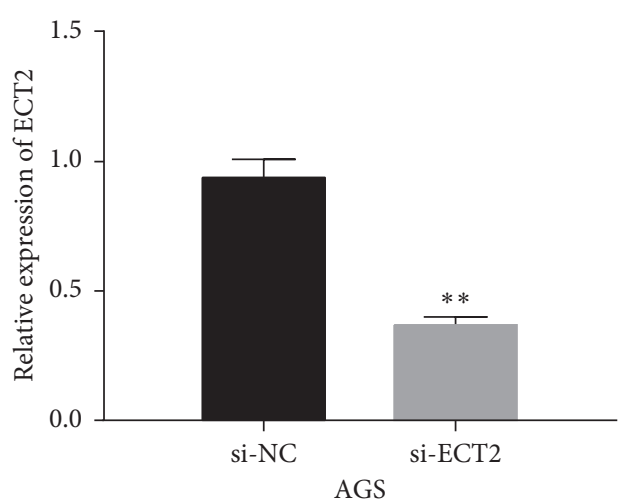

(b)

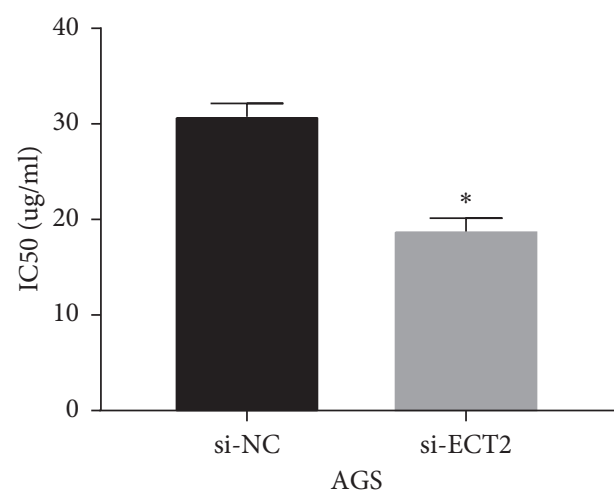

(d)

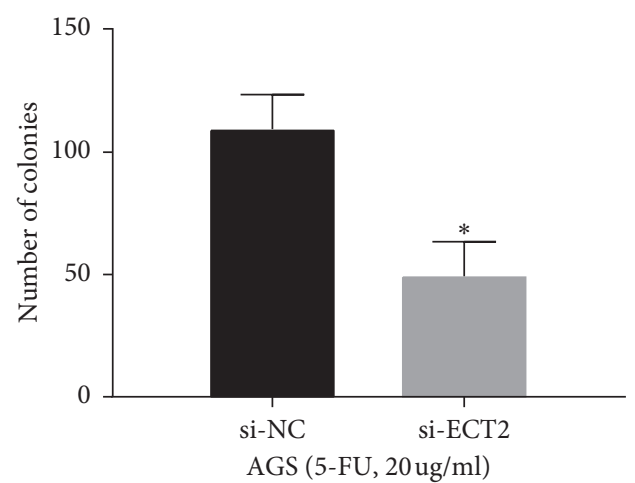

(e)

Figure 2: Continued. 

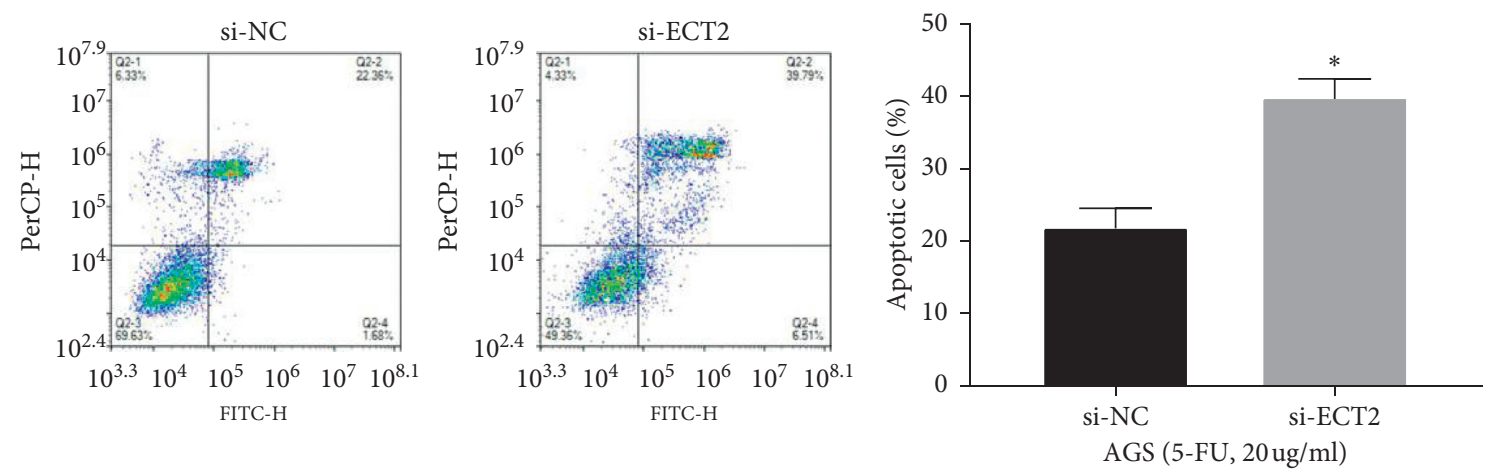

(f)

FIGURE 2: Knockdown of ECT2 enhancing 5-FU sensitivity in GC cells. (a) ECT2 expression in normal gastric mucosal cell line GES-1 and human GC cell lines AGS and NCI-N87. (b) ECT2 expression in AGS cells with si-ECT2 or si-NC. (c) Cell viability of si-NC and si-ECT2 with 5-FU treatment. (d) The IC50 value of AGS cells with si-NC and si-ECT2. (e) 5-FU sensitivity detection reconfirmed by the colony formation assay. (f) Apoptosis of AGS cells treated with 5-FU in si-NC and si-ECT2 groups. ${ }^{*} P<0.05 .{ }^{* *} P<0.01$.
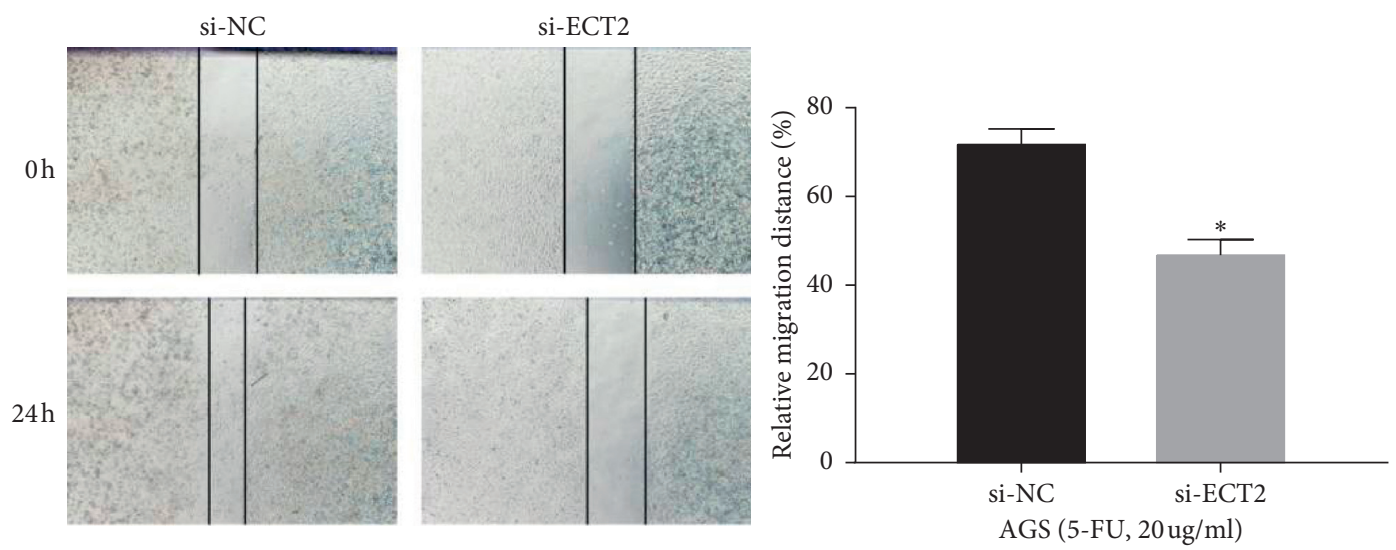

(a)

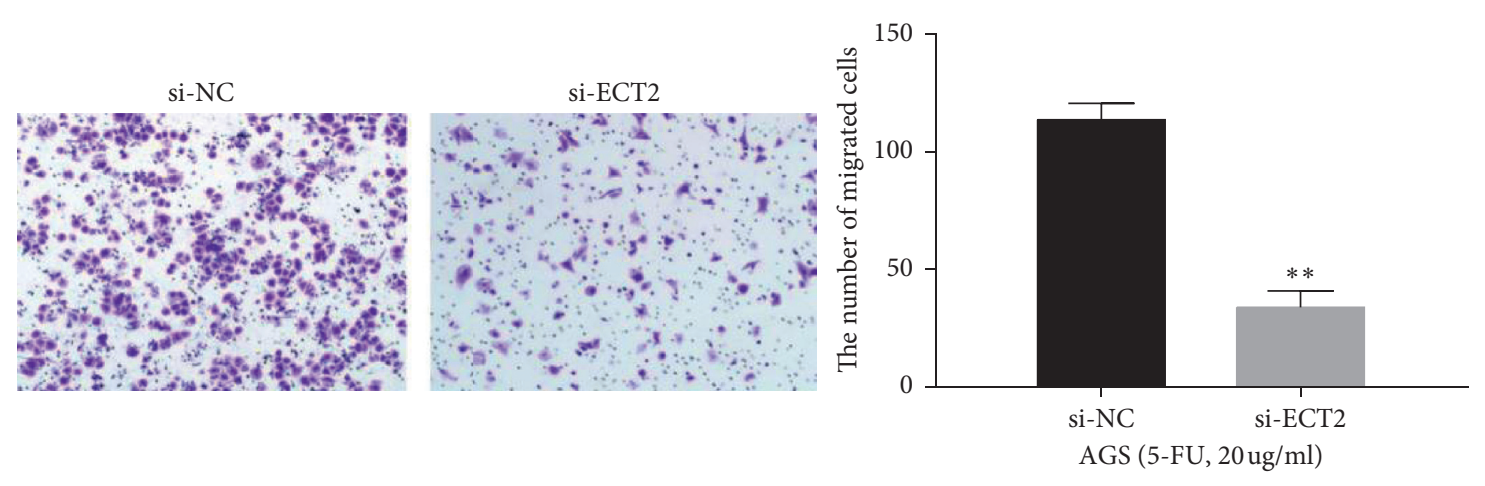

(b)

FIgURE 3: Continued. 


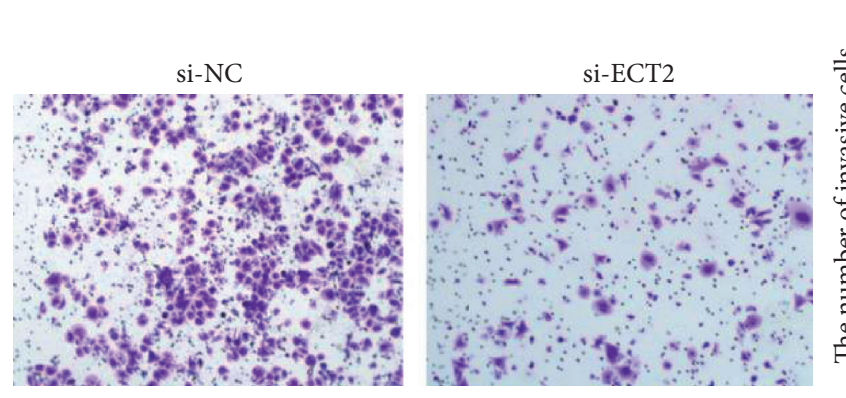

(c)

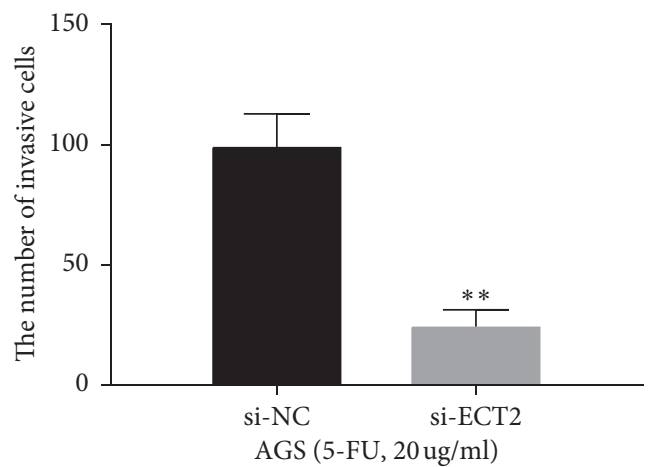

FIGURE 3: ECT2 silencing inhibiting the metastasis of GC cell lines treated by 5-FU. (a) The migration of AGS cells treated with 5-FU in siNC and si-ECT2 groups detected by the wound healing assay. (b, c) The migration and invasion of AGS cells treated with 5-FU in si-NC and si-ECT2 groups detected by the transwell assay. ${ }^{*} P<0.05 .{ }^{* *} P<0.01$.
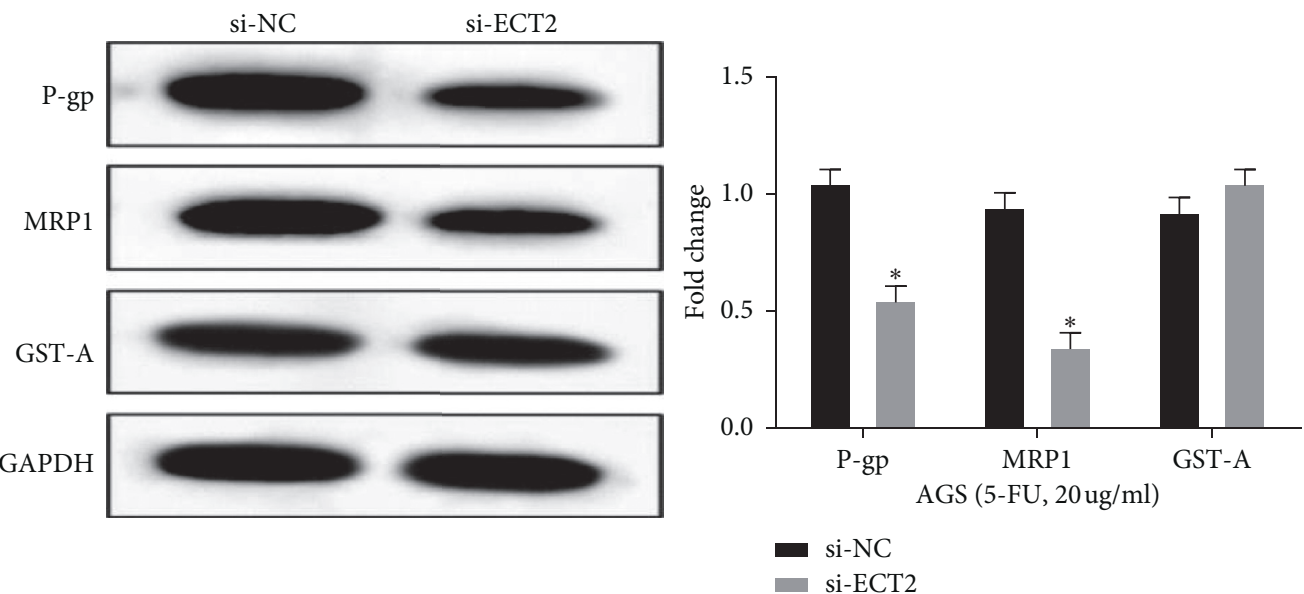

(a)
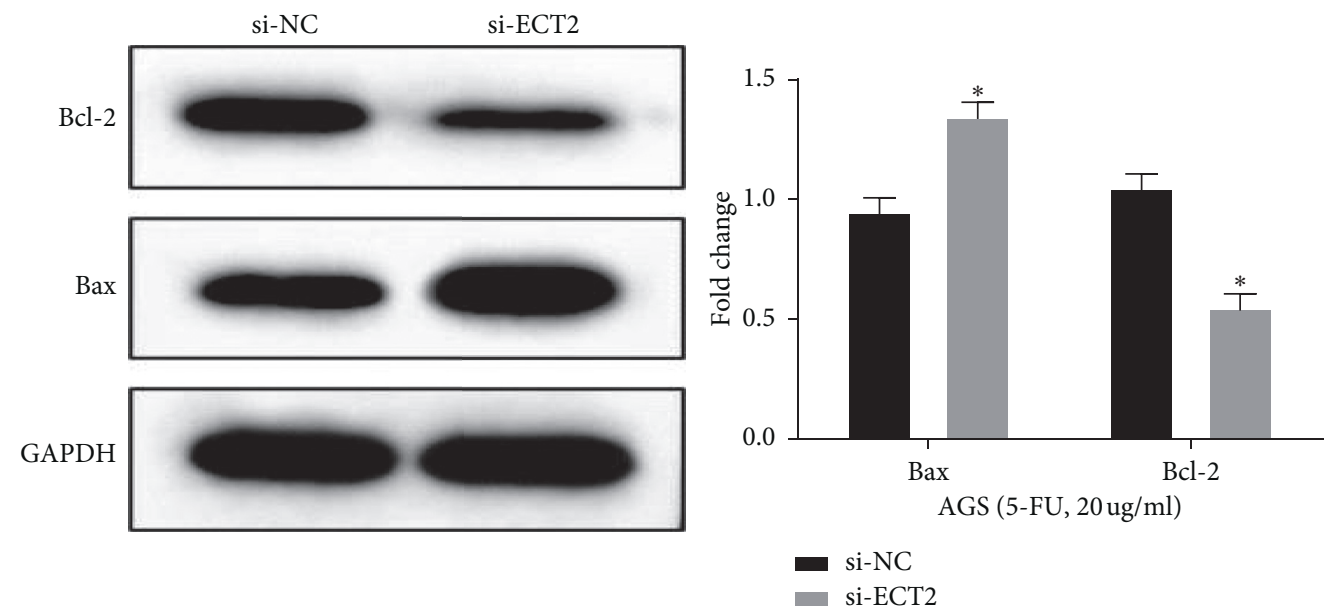

(b)

FIGURE 4: ECT2 downregulation increasing the sensitivity of GC cell lines to 5-FU by reducing the expressions of P-gp, MRP1, and Bcl-2. (a) Drug resistance-related proteins P-gp, MRP1, GST- $\pi$ detected in AGS cells treated with 5-FU in si-NC and si-ECT2 groups. (b) Apoptosis-related proteins Bcl-2 and Bax detected in AGS cells treated with 5-FU in si-NC and si-ECT2 groups. ${ }^{*} P<0.05$. 


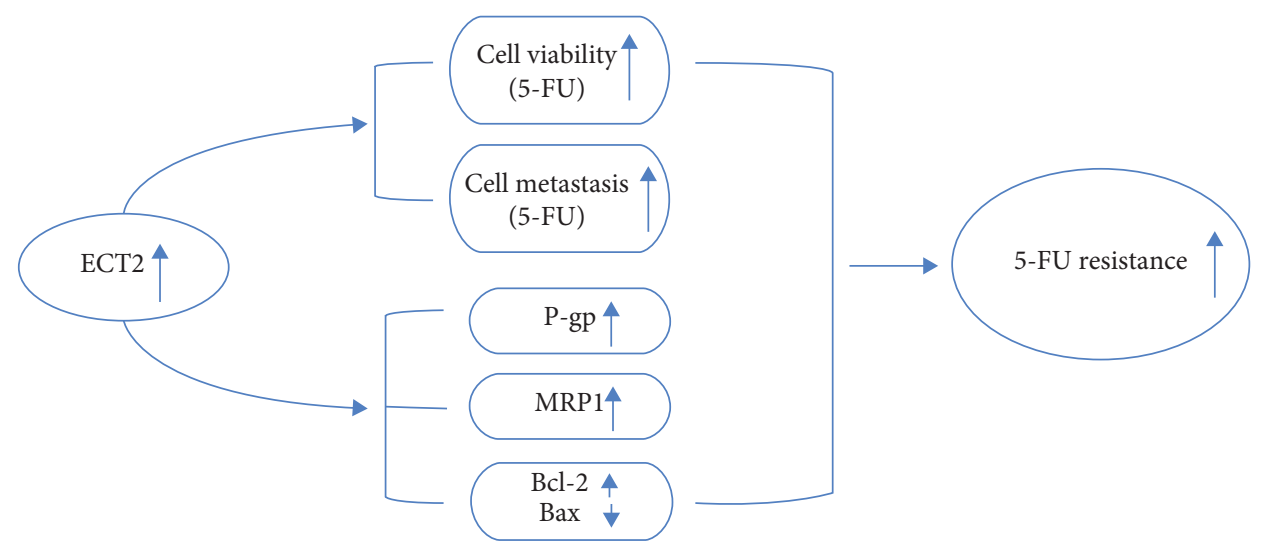

FIGURE 5: ECT2 affecting 5-FU sensitivity in GC cells by regulating cell viability, metastasis, and apoptosis-related proteins.

\section{Conclusion}

In conclusion, upregulation of ECT2 is observed in GC, which predicts adverse clinical outcomes in GC patients. Importantly, ECT2 knockdown attenuates 5-FU resistance in GC cells. Innovatively, we found that ECT2 may affect 5FU sensitivity in GC cells by regulating cell viability, metastasis, and apoptosis-related proteins. This study can provide a promising treatment option for GC patients.

\section{Data Availability}

The datasets used to support the findings of this study are available from the corresponding author upon request.

\section{Conflicts of Interest}

The authors declare that they have no conflicts of interest.

\section{References}

[1] M. Riihimäki, A. Hemminki, K. Sundquist, J. Sundquist, and K. Hemminki, "Metastatic spread in patients with gastric cancer," Oncotarget, vol. 7, no. 32, pp. 52307-52316, 2016.

[2] S. Ishaq and L. Nunn, "Helicobacter pylori and gastric cancer: a state of the art review," Gastroenterology and Hepatology from Bed to Bench, vol. 8, pp. S6-S14, 2015.

[3] R. L. Siegel, K. D. Miller, and A. Jemal, "Cancer statistics, 2019," CA: A Cancer Journal for Clinicians, vol. 69, no. 1, pp. 7-34, 2019.

[4] W. Chen, "Cancer statistics: updated cancer burden in China," Chinese Journal of Cancer Research, vol. 27, p. 1, 2015.

[5] A. Biagioni, I. Skalamera, S. Peri et al., "Update on gastric cancer treatments and gene therapies," Cancer and Metastasis Reviews, vol. 38, no. 3, pp. 537-548, 2019.

[6] F. Raza, H. Zafar, S. Zhang et al., "Recent advances in cell membrane-derived biomimetic nanotechnology for cancer immunotherapy," Advanced Healthcare Materials, vol. 10, no. 6, Article ID e2002081, 2021.

[7] F. Raza, H. Zafar, X. You, A. Khan, J. Wu, and L. Ge, "Cancer nanomedicine: focus on recent developments and self-assembled peptide nanocarriers," Journal of Materials Chemistry B, vol. 7, no. 48, pp. 7639-7655, 2019.

[8] H. Arai and T. E. Nakajima, "Recent developments of systemic chemotherapy for gastric cancer," Cancers, vol. 12, 2020.
[9] W.-J. Shi and J.-B. Gao, "Molecular mechanisms of chemoresistance in gastric cancer," World Journal of Gastrointestinal Oncology, vol. 8, no. 9, pp. 673-681, 2016.

[10] F. Raza, Y. Zhu, L. Chen et al., "Paclitaxel-loaded pH responsive hydrogel based on self-assembled peptides for tumor targeting," Biomaterials Science, vol. 7, no. 5, pp. 2023-2036, 2019.

[11] K. Nishibeppu, S. Komatsu, J. Kiuchi et al., "TRIM37 contributes to malignant outcomes and CDDP resistance in gastric cancer," Journal of Cancer, vol. 12, no. 2, pp. 316-325, 2021.

[12] Q. Ni, Y. Zhang, R. Tao, X. Li, and J. Zhu, "MicroRNA-95-3p serves as a contributor to cisplatin resistance in human gastric cancer cells by targeting EMP1/PI3K/AKT signaling," Aging, vol. 13, no. 6, pp. 8665-8687, 2021.

[13] H. Gao, J. Ma, Y. Cheng, and P. Zheng, "Exosomal transfer of macrophage-derived miR-223 confers doxorubicin resistance in gastric cancer," OncoTargets and Therapy, vol. 13, pp. 12169-12179, 2020.

[14] Z. J. An, Y. Li, B. B. Tan et al., "Up-regulation of KLF17 expression increases the sensitivity of gastric cancer to 5fluorouracil," International Journal of Immunopathology and Pharmacology, vol. 35, Article ID 20587384211010925, 2021.

[15] X. Wang, Z. Tong, and H. Liu, "MiR-223-3p targeting epithelial cell transforming sequence 2 oncogene inhibits the activity, apoptosis, invasion and migration of MDA-MB-468 breast cancer cells," OncoTargets and Therapy, vol. 12, pp. 7675-7684, 2019.

[16] J. Gao, C. Dai, X. Yu, X. B. Yin, and F. Zhou, "Upregulated microRNA-194 impairs stemness of cholangiocarcinoma cells through the Rho pathway via inhibition of ECT2," Journal of Cellular Biochemistry, vol. 121, no. 10, pp. 4239-4250, 2020.

[17] Y. Yu, O. Cai, P. Wu, and S. Tan, "MiR-490-5p inhibits the stemness of hepatocellular carcinoma cells by targeting ECT2," Journal of Cellular Biochemistry, vol. 120, no. 1, pp. 967-976, 2019.

[18] T. Zhi, K. Jiang, X. Xu et al., "ECT2/PSMD14/PTTG1 axis promotes the proliferation of glioma through stabilizing E2F1," Neuro-Oncology, vol. 21, no. 4, pp. 462-473, 2019.

[19] J. Xie, P. Lei, and Y. Hu, "Small interfering RNA-induced inhibition of epithelial cell transforming sequence 2 suppresses the proliferation, migration and invasion of osteosarcoma cells," Experimental and Therapeutic Medicine, vol. 9, no. 5, pp. 1881-1886, 2015.

[20] D. Xu, Y. Wang, J. Wu et al., "ECT2 overexpression promotes the polarization of tumor-associated macrophages in 
hepatocellular carcinoma via the ECT2/PLK1/PTEN pathway," Cell Death \& Disease, vol. 12, no. 2, p. 162, 2021.

[21] J. Liu, M. Zhou, Y. Ouyang, L. Du, L. Xu, and H. Li, "Identification of potential biomarkers and their clinical significance in gastric cancer using bioinformatics analysis methods," PeerJ, vol. 8, Article ID e9174, 2020.

[22] H. Wang, H. Liu, J. Li et al., "Effect of Ect2 expression on the growth of triple-negative breast cancer cells with paclitaxel intervention," OncoTargets and Therapy, vol. 13, pp. 1290512918, 2020.

[23] Y. Chen, P. Tian, and Y. Liu, "P53 and protein phosphorylation regulate the oncogenic role of epithelial cell transforming 2 (ECT2)," Medical Science Monitor, vol. 23, pp. 3154-3160, 2017.

[24] Y. Jin, Y. Yu, Q. Shao et al., "Up-regulation of ECT2 is associated with poor prognosis in gastric cancer patients," International Journal of Clinical and Experimental Pathology, vol. 7, pp. 8724-8731, 2014.

[25] D. Y. Gong, X. Chen, T. L. Yang et al., "Upregulation of ECT2 is associated with transcriptional program of cancer stem cells and predicts poor clinical outcome in gastric cancer," Oncology Letters, vol. 20, p. 54, 2020.

[26] H.-B. Wang, H.-C. Yan, and Y. Liu, "Clinical significance of ECT2 expression in tissue and serum of gastric cancer patients," Clinical and Translational Oncology, vol. 18, no. 7, pp. 735-742, 2016.

[27] K. Zhou, H. Mai, S. Zheng et al., "OTUB1-mediated deubiquitination of FOXM1 up-regulates ECT-2 to promote tumor progression in renal cell carcinoma," Cell \& Bioscience, vol. 10, no. 1 , p. 50, 2020.

[28] C. Li, Z. Peng, Y. Wang et al., "Epithelial cell transforming 2 is regulated by yes-associated protein 1 and mediates pancreatic cancer progression and metastasis," American Journal of Physiology-Gastrointestinal and Liver Physiology, vol. 320, no. 3, pp. G380-G395, 2021.

[29] H. Zhang, H. Jiang, H. Zhang, J. Liu, X. Hu, and L. Chen, "Ribophorin II potentiates P-glycoprotein- and ABCG2mediated multidrug resistance via activating ERK pathway in gastric cancer," International Journal of Biological Macromolecules, vol. 128, pp. 574-582, 2019.

[30] B. Tan, Y. Li, Q. Zhao, L. Fan, and D. Wang, "ZNF139 increases multidrug resistance in gastric cancer cells by inhibiting miR-185," Bioscience Reports, vol. 38, 2018.

[31] L. Singh, N. Pushker, N. Saini et al., "Expression of pro-apoptotic Bax and anti-apoptotic Bcl-2 proteins in human retinoblastoma," Clinical \& Experimental Ophthalmology, vol. 43, no. 3, pp. 259-267, 2015. 